\title{
Limits on the network sensitivity function for homogeneous multi-agent systems on a graph
}

\author{
Stefania Tonetti and Richard M. Murray
}

\begin{abstract}
This paper explores some tradeoffs and limits of performance in feedback control of interconnected multiagent systems, focused on the network sensitivity functions. We consider the interaction topology described by a directed graph and we prove that the sensitivity transfer functions between every pair of agents, arbitrarily connected, can be derived using a version of Mason's direct rule. An analysis of the role of cycles shows that these structures influence and considerably limit the behavior of the system. We also derive a networked version of Bode's integral formula, showing that it still holds for multi-agent systems.
\end{abstract}

\section{INTRODUCTION}

In recent years, thanks to advances in technology, attention has been focused on the control of distributed dynamic systems. In numerous mission scenarios, the concept of a group of agents cooperating to achieve a determined goal is very attractive when compared with the solution of one single vehicle. In this class of systems, even if the agents are dynamically decoupled, they are coupled through the common task they have to achieve. When the number of agents grows, centralized control is no longer feasible and distributed control techniques became necessary. Applications of coordinated control of multiple vehicles can be found in many fields, including microsatellite clusters, formation flying of unmanned aerial vehicles, automated highway systems and mobile robotics.

The problem of distributed control has been widely studied with tools from graph theory [1], [2], [3]. We consider agents with identical linear dynamics and we model the interconnection topology as a graph, in which the single agents are represented by a vertex, while the interaction links are the arcs.

The distributed control problem has been handled in different ways and with different tools: dissipative theory and linear matrix inequalities [4], edge agreement [5], [6], linear quadratic regulator [7], decomposition and linear matrix inequalities [8]. In almost all the works mentioned above the control is applied to undirected graphs. If the graph is undirected the problem becomes easier because all the matrices associated with the graph, like the Laplacian, are symmetric.

One approach to distributed control is to use leaderfollower arrangement. This approach is well studied and

This work was not supported by any organization

$\mathrm{S}$. Tonetti is with the Department of Aerospace Engineer, Politecnico di Milano, Milano, Italy tonettidaero.polimi.it

R. M. Murray is with the Department of Control and Dynamical Systems, California Institute of Technology, Pasadena, CA 91125 USA murraydcds.caltech.edu representative papers exploring graph-theoretic ideas in the context of a leader-follower architecture include [3] and [9], where a double-graph control strategy was proposed. This topology represents a particular case, where the leader has a more important role than the other agents and this may not always be desirable.

The importance of the cycles in distributed control has already been pointed out in several past works: Zelazo et al. [5], [6] investigated the role of cycles and trees in the edge Laplacian for the edge agreement problem, while Fax and Murray [10] derived a relation between the presence of cycles and the stability of the formation.

The contribution of this paper is to show a general method to derive the transfer functions between any pair of agents, where the interconnection topology is described by arbitrary directed graphs with the leader-follower architecture is as a particular case. We do not present a formula that simplifies the complexity of computing sensitivity matrix, but we give insight into its structure, in order to better understand the role that cycles and paths play. We then analyze mechanisms that rule the behavior of a multi-agent system and we show intrinsic limits on the controller design due to the topology.

The paper is organized as follows. In section II we briefly review the principal concepts of graph theory and the main stability results on formation control. Section III extends several classical control concepts in order to deal with multiagent systems. The core of the paper is presented in Section IV. In Section V some design considerations and limitations are proposed. Finally, Section VI contains examples on different interaction topologies and the conclusions of the paper are reported in Section VII.

A more detailed version of the paper is available as a technical report [11].

\section{PRELIMINARIES}

In this section we summarize some of the key concepts and definitions from graph theory that will be used in the paper. A more detailed presentation of graph theory can be found in [12].

A directed graph $\mathcal{G}$ is a set of vertices or nodes $V$ and a set of $\operatorname{arcs} A \subset V^{2}$ whose elements $a=(u, v) \in A$ characterize the relation between distinct pairs of vertices $u, v \in V$. For an $\operatorname{arc}(u, v)$ we call $u$ the tail and $v$ the head. The in(out)degree of a vertex $v$ is the number of arcs with $v$ as its head (tail). A directed path in a graph is a sequence of vertices such that from each of its vertices there is an arc to the next vertex in the sequence. A directed path with no repeated vertices is called a simple directed path. 
A directed graph is called strongly connected if there is a directed path from each vertex in the graph to every other vertex. A directed graph is weakly connected if every vertex can be reached from every other but not necessarily following the directions of the arcs. A complete directed graph is a graph where each pair of vertices has an arc connecting them. A simple cycle is a closed path that is self-avoiding (does not revisit nodes, other than the first). A acyclic directed graph is a directed graph without cycles. A star graph of order $N$, sometimes simply known as an $N$-star, is a graph on $N$ nodes with one node having degree $N-1$ and the other $N-1$ nodes having degree 1 .

The structure of a graph can be described by appropriate matrices. The adjacency matrix $\mathcal{A}$ of a graph $\mathcal{G}$ is a square matrix of size $|V|$, defined by $\mathcal{A}_{i j}=1$ if $(i, j) \in A$, and zero otherwise. The normalized Laplacian matrix $\mathcal{L}$ of a directed graph $\mathcal{G}$ is a square matrix of size $|V|$, defined by $\mathcal{L}_{i j}=1$ if $i=j, \mathcal{L}_{i j}=1 / d_{i}$ if $(i, j) \in A$, where $d_{i}$ is the outdegree of the $i$ th vertex, $\mathcal{L}_{i j}=0$ otherwise. We can observe that if $d_{i}>0$ for all vertices in the graph, $\mathcal{L}$ has zero row sum, which implies that zero is an eigenvalue of $\mathcal{L}$. Furthermore if $\mathcal{G}$ is strongly connected, zero is a simple eigenvalue of $\mathcal{L}$ and all eigenvalues of $\mathcal{L}$ lie in a disk in the complex plane with unity radius and centered at $1+0 j$ [13].

We consider a formation of $N$ agents with identical linear dynamics. The normalized Laplacian matrix $\mathcal{L}$ of the graph is used to represent the interaction topology. Suppose each individual agent is a SISO system with local loop composed of a local controller $C(s)$ and a plant model $P(s)$. According to Fax [10], the multi-agent system is stable if and only if the net encirclement of the critical points $-\lambda_{i}^{-1}(\mathcal{L})$ by the Nyquist plot of $P(s) C(s)$ is zero for all nonzero $\lambda_{i}(\mathcal{L})$, where $\lambda_{i}(\mathcal{L})$ are the eigenvalues of the normalized Laplacian matrix $\mathcal{L}$ of the graph.

We will utilize some additional notation used by Fax [10]. The Kronecker product $\otimes$ between two matrices $P=\left[p_{i j}\right]$ and $Q=\left[q_{i j}\right]$ is defined as $P \otimes Q=\left[p_{i j} Q\right]$. This is a block matrix with the $i j$ th block of $p_{i j} Q$. Let $I_{n}$ indicate the identity matrix of order $n$. To represent the matrix $M$ repeated $N$ times along the diagonal we write $\widehat{M}=I_{N} \otimes M$. Letting $n$ be the number of configuration (output) variables of each agent that can be controlled, $\mathcal{L}_{(n)}$ is of dimension $N n \times N n$, i.e. $\mathcal{L}_{(n)}=\mathcal{L} \otimes I_{n}$.

\section{STABILITY AND PERFORMANCE MEASURE}

In this section we will investigate how the performance specifications for single agent control systems translate into requirements for multi-agent systems.

We consider the multi-agent feedback system in Figure 1 , where $\mathbf{r} \in R^{N}$ is the vector of the reference signals of each agent, $\mathbf{e} \in R^{N}$ are the errors between $\mathbf{r}$ and the process outputs $\mathbf{y} \in R^{N}, \mathbf{u} \in R^{N}$ is the control signal vector and $\mathbf{d} \in R^{N}$ and $\mathbf{n} \in R^{N}$ are the load disturbances and the measurement noises respectively. Define the networked loop transfer function matrix as $\widehat{L}(s)=\widehat{P}(s) \widehat{C}(s)$. All through the paper we will consider only stable systems. The relations between the inputs and the interesting signals of the system

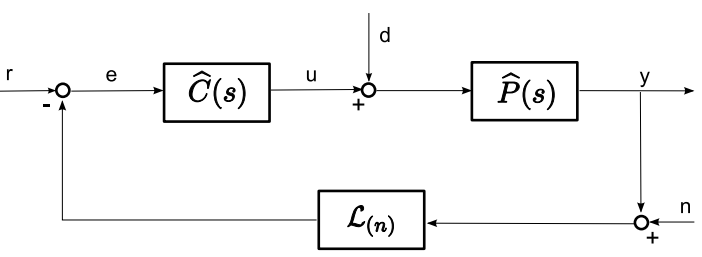

Fig. 1. Block diagram of a multi-agent feedback system.

are given by the following transfer function matrices that can be recognized to be the networked version of the single agent ones. We define the networked sensitivity function matrix $\widetilde{S}(s)$ as

$$
\widetilde{S}(s)=\left(I+\mathcal{L}_{(n)} \widehat{P}(s) \widehat{C}(s)\right)^{-1},
$$

the networked complementary sensitivity function matrix $\widetilde{T}(s)=\widetilde{S}(s) \widehat{P}(s) \widehat{C}(s)$, the networked load sensitivity function matrix $\widehat{P}(s) \widetilde{S}(s)$ and the networked noise sensitivity function matrix $\mathcal{L}_{(n)} \widehat{C}(s) \widetilde{S}(s)$.

From now on, without loss of generality, we will consider $n=1$ so that each agent has a single output variable that is being controlled. In analogy with the single agent case, in order to guarantee stability, robustness and good performance, we want to have

$|\widetilde{S}(j \omega)| \ll 1$ for $\omega \ll \omega_{c}$, and $|\widetilde{S}(j \omega)| \approx 1$ for $\omega \gg \omega_{c}$

$|\widetilde{T}(j \omega)| \ll 1$ for $\omega \gg \omega_{c}$, and $|\widetilde{T}(j \omega)| \approx 1$ for $\omega \ll \omega_{c}$.

Since in a multi-agent control the critical point for the stability of the system is no longer the point -1 , but the collection of points $-\lambda_{i}^{-1}(\mathcal{L})$, the well-know indicators for how near the Nyquist plot is to the critical points need to be redefined.

Define the networked gain margin $G M_{n}$ as the minimum scaling that will cause the Nyquist curve for $L(j \omega)$ intersect one of the eigenvalues of $\mathcal{L}$. Define the networked phase margin $P M_{n}$ as the minimum angle between the argument of $-\lambda^{-1}$ and $L\left(j \omega_{c_{\lambda}}\right)$, where $\omega_{c_{\lambda}}$ is the angular frequency where the Nyquist plot intersects the circle with radius $\left|-\lambda^{-1}\right|$ closest to the point $-\lambda^{-1}$, i.e. where $|L(j \omega)|=\left|-\lambda^{-1}\right|$.

\section{DISTURBANCE REJECTION FOR MULTI-AGENT SYSTEMS}

In this section we show how to derive the networked sensitivity transfer functions between any pair of agents for a given topology. We are dealing with determining transfer functions on graphs and an effective and straightforward means to achieve it is signal-flow graph theory. The main results in this area are due to Mason [14], who derived a rule to compute the transfer function of a signal-flow graph, commonly known as Mason's direct rule [15], [16].

We define the Laplacian weight of a simple directed path of length $k$ from $i$ to $j$, where $i=i_{0}, i_{1}, \ldots, i_{k}=j$, as the product of the negative inverse of the outdegrees $d$ of all the nodes in the path besides the last one:

$$
\mathcal{L} w_{i_{0} i_{k}}^{k}:=\operatorname{sgn}(k) \prod_{t=0}^{t=k-1}\left(-\frac{1}{d_{i_{t}}}\right),
$$


where $\operatorname{sgn}(k)=-1$ if $k$ is odd, $\operatorname{sgn}(k)=+1$ if $k$ is even. We say a path is a degenerate path if it is a path of length zero between a node and itself and we define its Laplacian weight as one: $\mathcal{L} w_{i i}^{0}=1$. Since in a cycle every node can be the starting and ending node, the Laplacian weight of a cycle will be indicated with $o$ as subscript. The Laplacian weight of a cycle of length $k$ will be

$$
\mathcal{L} w_{o}^{k}:=\operatorname{sgn}(k-1) \prod_{t=0}^{t=k-1}\left(-\frac{1}{d_{i_{t}}}\right), i_{0}=i_{k},
$$

We define disjoint cycles in $\mathcal{G}$ to be a set of non-adjacent simple cycles, that is, two simple cycles that do not share any common nodes. The length of disjoint cycles is given by the sum of the lengths of the composing simple cycles, while the Laplacian weight of disjoint cycles is given by the product of the Laplacian weights of the composing simple cycles.

Define $\mathcal{G}_{i j}^{k}$ as the subgraph of $\mathcal{G}$ obtained from $\mathcal{G}$ by removing all the nodes and all the arcs touching the simple directed path from node $i$ to node $j$ of length $k$.

Theorem 1: The sensitivity transfer function between every pair of nodes $i$ and $j$ of a generic graph $\mathcal{G}$ can be derived using the following expression, which is a version of the Mason's Direct Rule:

$$
\widetilde{S}_{i j}=\frac{1}{\Delta} \sum_{\text {paths } p \in \mathcal{G}} T_{p} \Delta_{p}
$$

where:

1) $\Delta$ is the determinant of $(I+\mathcal{L} \widehat{P} \widehat{C})$,

$$
\begin{aligned}
\Delta= & (1+P C)^{N} \\
& +\sum_{\text {cycles } o \in \mathcal{G}}\left(\mathcal{L} w_{o}^{k}\right)(1+P C)^{(N-k)}(P C)^{k} ;
\end{aligned}
$$

2) $T_{p}$ is the 'gain' of the $p^{t h}$ simple directed path from node $i$ to node $j$ of length $k$,

$$
T_{p}=\left(\mathcal{L} w_{i j}^{k}\right)(P C)^{k}
$$

and

3) $\Delta_{p}$ is the value of $\Delta$ for the subgraph $\mathcal{G}_{i j}^{k}$ not touching the $p^{t h}$ simple directed path from node $i$ to node $j$ of length $k$,

$$
\begin{aligned}
& \Delta_{p}=(1+P C)^{(N-1-k)} \\
& +\sum_{\text {cycles } o \in \mathcal{G}_{i j}^{k}}\left(\mathcal{L} w_{o}^{\bar{k}}\right)(1+P C)^{(N-1-k-\bar{k})}(P C)^{\bar{k}},
\end{aligned}
$$

and $\bar{k}$ represents the length of the cycles in $\mathcal{G}_{i j}^{k}$.

The proof can be found in [11].

In classical control theory, in order to attenuate the disturbances entering the system, the gain of $S$ is reduced at low frequencies and consequently the gain of the open loop transfer function is large at those frequencies. Therefore it is interesting to study the asymptotic behavior of the networked sensitivity functions for $|P C| \rightarrow \infty$.

The denominator in equation (3) is the determinant of $(I+\mathcal{L} \widehat{P} \widehat{C})$, so it is a polynomial of $N$ th order in $P C$. It depends only on the cycles in $\mathcal{G}$ and it is the same for all the $\widetilde{S}_{i j}$ and $\widetilde{S}_{i i}$.

Proposition 2: Given a graph $\mathcal{G}$, the determinant of the normalized Laplacian matrix $\mathcal{L}$ is

$$
\operatorname{det}(\mathcal{L})=1+\sum_{\text {cycles } o \in \mathcal{G}}\left(\mathcal{L} w_{o}^{k}\right)
$$

Proof: We will follow the proof of Theorem 1 in [11]. Consider the transformed graph $\widetilde{\mathcal{G}}$ with the same topology described by $\mathcal{L}$ but the weight of each arc equal to

$$
w_{i j}=\frac{1}{d_{i}}, \forall(i, j) \in \widetilde{\mathcal{G}}
$$

and no self-loops $\left(w_{i i}=0\right)$. The transformed weighted adjacency matrix $\widetilde{\mathcal{A}}$ for the graph $\widetilde{\mathcal{G}}$ will be $\widetilde{\mathcal{A}}=I-\mathcal{L}$ and the transformed gain matrix $\widetilde{\mathcal{M}}=(I-I+\mathcal{L})^{-1}=(\mathcal{L})^{-1}$. The denominator of the gain matrix is the determinant of $\mathcal{L}$.

Theorem 3: If every vertex in $\mathcal{G}$ has outdegree greater than zero, the coefficient of $(P C)^{N}$ in the complete polynomial expression of the denominator is always zero:

$$
1+\sum_{\text {cycles } o \in \mathcal{G}}\left(\mathcal{L} w_{o}^{k}\right)=0
$$

Proof: The coefficient of $(P C)^{N}$ can be computed from equation (4) and gives the left hand side of equation (8). This term is $\operatorname{det}(\mathcal{L})$ by Proposition 2 . For graphs with $d_{i}>0, \forall i$ we know that $\mathcal{L}$ has a zero eigenvalue and $\operatorname{so} \operatorname{det}(\mathcal{L})=0$.

Therefore for weakly connected graphs with outdegree of every node greater than zero, the polynomial in the denominator is order $N-1$ in $P C$. The asymptotic value as $|P C| \rightarrow \infty$ depends on the coefficient of $(P C)^{(N-1)}$ and it is easy to show it is given by

$$
N+\sum_{\text {cycles } o \in \mathcal{G}}\left(\mathcal{L} w_{o}^{k}\right)(N-k) .
$$

If a graph has at least one node with outdegree equal to zero, the Laplacian matrix looses its property of zero row sum and $\operatorname{det}(\mathcal{L}) \neq 0$. Graphs of this type will have a polynomial in the denominator of $N$ th order.

The numerator of $\widetilde{S}_{i j}$ is an element of the adjugate matrix (the transpose of the cofactors matrix) of $(I+\mathcal{L} \widehat{P} \widehat{C})$ and it is a polynomial of order $N-1$ in $P C$. The coefficients depend on all the simple directed paths from node $i$ to node $j$ and on the cycles of the subgraphs $\mathcal{G}_{i j}^{k}$. The value of the coefficient of $(P C)^{(N-1)}$ in the complete polynomial expression of the numerator for $i \neq j$ is given by

$$
\sum_{\text {paths } i j \in \mathcal{G}}\left(\left(\mathcal{L} w_{i j}^{k}\right) \cdot\left(1+\sum_{\text {cycles } o \in \mathcal{G}_{i j}^{k}}\left(\mathcal{L} w_{o}^{\bar{k}}\right)\right)\right) \text {. }
$$

If no cycles exist in $\mathcal{G}_{i j}^{k}$, then $\mathcal{L} w_{o}^{\bar{k}}=0$. If no path exists from node $i$ to node $j, \widetilde{S}_{i j}$ will be always zero for every $|P C|$ value.

If $i=j$ we have a degenerate path and $\mathcal{L} w_{i i}^{0}=1$. The subgraph $\mathcal{G}_{i j}^{k}$ is obtained by removing the $i$ th node and all the arcs with head or tail in $i$ and it will be indicated by $\mathcal{G}_{i}$. 
TABLE I

SAMPLE GRAPHS AND NETWORKED SENSITIVITY FUNCTIONS

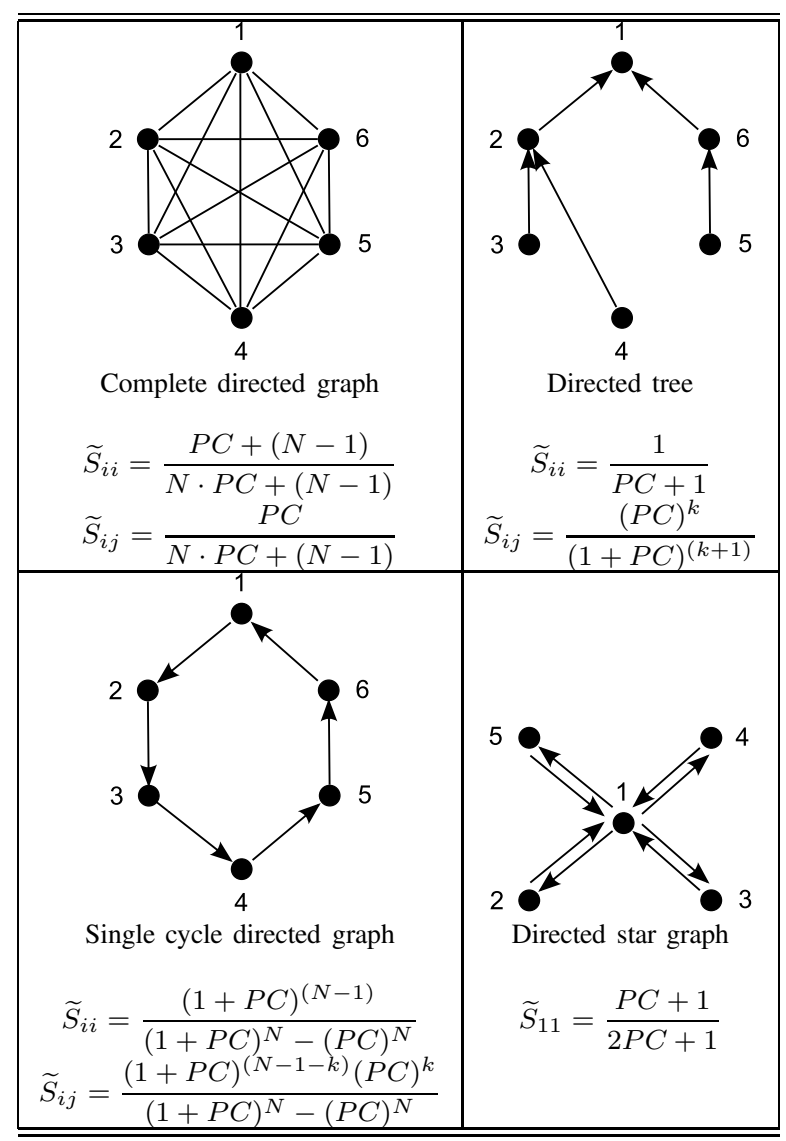

We have only to look at the Laplacian weights $\mathcal{L} w_{o}$ of all the simple cycles or disjoint cycles in $\mathcal{G}_{i}$. Equations (5) and (6) for $i=j$ simplify to

$$
+\sum_{\text {cycles } o \in \mathcal{G}_{i}}^{(1+P C)^{(N-1)}}\left(\mathcal{L} w_{o}^{\bar{k}}\right)(1+P C)^{(N-1-\bar{k})}(P C)^{\bar{k}},
$$

and the value of the coefficient of $(P C)^{(N-1)}$ in the complete polynomial expression of the numerator for $i=j$ becomes

$$
1+\sum_{\text {cycles } o \in \mathcal{G}_{i}}\left(\mathcal{L} w_{o}^{\bar{k}}\right) .
$$

From Theorem 3 we can assert that if every node has outdegree greater than zero, both $\widetilde{S}_{i j}$ and $\widetilde{S}_{i i}$ are proper functions in terms of the open loop transfer function. If at least one node has $d=0, \widetilde{S}_{i j}$ and $\widetilde{S}_{i i}$ are strictly proper functions.

To better understand (1)-(12), we consider some sample graphs. In Table I we summarize the networked sensitivity functions for special graphs. A more detailed analysis and all the proofs can be found in [11]. In all the examples no self-loops have been considered.

\section{DESIGN CONSIDERATIONS}

In the previous sections we have shown how to derive all the sensitivity transfer functions given a topology. Now we will analyze how to design the topology in order to achieve, when possible, desired levels of performance and we will present some design limitations. Furthermore the role of the cycles will be discussed more in detail.

To aid in our designs, we would like to find a relationship between $\widetilde{S}_{i i}$ and $\widetilde{S}_{i j}$. For low loop gains we have

$$
\lim _{|P C| \rightarrow 0}\left|\widetilde{S}_{i i}\right|=1 \text { and } \lim _{|P C| \rightarrow 0}\left|\widetilde{S}_{i j}\right|=0 .
$$

Theorem 4: When the open loop gain is very high, the magnitude of the networked sensitivity function between every node and node $i$ reaches the same value:

$$
\left|\widetilde{S}_{j i}\right| \approx\left|\widetilde{S}_{i i}\right|,|P C| \rightarrow \infty .
$$

The proof can be found in [11].

Equation (13) states that for very high gain of the system, the disturbance affecting agent $i$ is propagated with the same intensity through all its neighbors.

Analyzing the signs of the Laplacian weights we can observe the following: $\left(\mathcal{L} w_{i j}\right)$ is always positive, for simple cycles $\left(\mathcal{L} w_{o}\right)$ is always negative and for disjoint cycles nothing can be said about the sign of $\left(\mathcal{L} w_{o}\right)$. As it is defined, $\left|\mathcal{L} w_{o}\right| \leq 1$ and the more cycles there are in the subgraph, the more negative it is.

Define the global loopiness $C$ as the total number of distinct simple cycles in the graph (cyclic permutations of the nodes do not count). The local counterpart, $C^{(i)}$, is the number of simple cycles that pass through node $i$. Define the loopiness ratio of a node as $C_{r}^{(i)}=C^{(i)} / C$.

The asymptotic value of the sensitivity depends on the loopiness ratio. We have that the lower $C_{r}^{(i)}$ is, the lower the asymptotic value of $\widetilde{S}_{i i}$ and $\widetilde{S}_{j i}$ will be. This is because $\left(\mathcal{L} w_{o}\right)$ is always negative and we would like to have the highest number of cycles in the subgraph $\mathcal{G}_{i}$, in order to keep the asymptotic value as low as possible. If a small number of cycles pass through node $i$ compared to the total number of cycles in the graph, in the subgraph $\mathcal{G}_{i}$ there will be a large number of cycles left and then the asymptotic value will be low. In a directed star graph (see Table I) the central node has the worst loopiness ratio, $C_{r}=1$, because all the cycles of the graph are concentrated in it, while all the other nodes have a good loopiness ratio because only one cycle passes through them.

Unfortunately we cannot bring all the asymptotic values to be small at the same time because all the asymptotic values of $\widetilde{\mathcal{S}}_{i i}$ sum up to the unity. If we look at the sum of all the asymptotic values of the numerator of $\widetilde{\mathcal{S}}_{i i}(12)$ for graph with $d_{i}>0, \forall i \in \mathcal{G}$, we can see that they sum up to the asymptotic value of the denominator (4). This is because a cycle is not counted in $\mathcal{G}_{i}$ if the node $i$ belongs to the cycle, therefore each cycle in all the $\mathcal{G}_{i}$ is counted $(N-k)$ times. It implies that there are fundamental limitations to what can be achieved by control and that control design can be viewed as a redistribution of disturbance attenuation at low frequencies 
among the agents. Thus if we want to keep all the asymptotic values as small as possible, the best result we can achieve is $1 / N$ for all the nodes, like in complete directed graphs and single cycle directed graphs. In order to obtain the same result in a generic graph, we have to equally distribute the cycles on the nodes, like in a regular graph. The loopiness ratio should be more or less the same for all the nodes. The worst sensitivity function we can have is that of a directed star graph.

We have seen that relation (8) holds only if every node has outdegree greater than zero. By definition in a leaderfollower topology the leader node has $d=0$, leading it to have a sensitivity transfer function where the degree of the numerator is less than the degree of the denominator. Therefore any leader-follower topology has the asymptotic value of any sensitivity function equal to zero for $|P C| \rightarrow \infty$. The diagonal sensitivity transfer function of an agent with outdegree equal to zero, will be in any case always equal to $S$. This is because since that agent node is not involved in any cycle, $\mathcal{G}$ and $\mathcal{G}_{i}$ will have exactly the same cycles, and (11) and (4) will differ only by a $(1+P C)$ at the denominator.

Bode [17] showed that for a SISO, stable open loop system $P(s) C(s)$, the integral of $\log (|S(j \omega)|$ is a constant value and it is equal to zero.

Theorem 5: In a multi-agent system, Bode's integral formula for stable open loop systems still holds for each diagonal interconnected sensitivity function $\widetilde{S}_{i i}$, no matter what the interconnection topology is:

The proof can be found in [11].

$$
\int_{0}^{\infty} \log \left(\left|\widetilde{S}_{i i}(j \omega)\right|\right) d \omega=0 .
$$

\section{EXAMPLES}

In this section we apply the theory developed above to some formations and we analyze the frequency domain behavior.

Suppose there are five agents in the formation with identical dynamics $P(s)=1 /\left(s^{2}+s+4\right)$ and the local controller $C(s)=(800 s+2000) /(s+40)$. It can be shown that $C(s)$ is a stabilizing controller for a single agent with infinite gain margin and $60^{\circ}$ of phase margin. The magnitude for very low frequencies is $|S|=7.4 \cdot 10^{-2}$.

Let us see now what happens when we have the multiagent system. In the following examples we will consider only stable topologies and we will focus on performance. Suppose we have the two interaction topologies shown in Figure 2 .

Topology 1 represents a leader-follower scheme, where agent 1 is the leader and the others are followers. Using the fact that for low frequencies $\left|\widetilde{S}_{j i}\right| \approx\left|\widetilde{S}_{i i}\right|$ we write the diagonal sensitivity functions. We already know that $\widetilde{S}_{11}=S$, while the others $\widetilde{S}_{i i}$ are all equal and they can be computed with equation (3). The Bode plot is shown in Figure 3. As we expected, for low and high frequencies the leader-follower sensitivity functions behave like the single agent one. But for frequencies near the cut-off frequency, the followers have a high peak value of about $M_{s}=5$, meaning

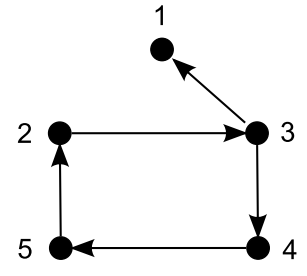

(a) Topology 1

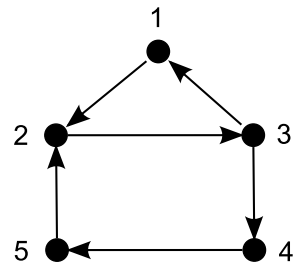

(b) Topology 2
Fig. 2. Two different topologies.

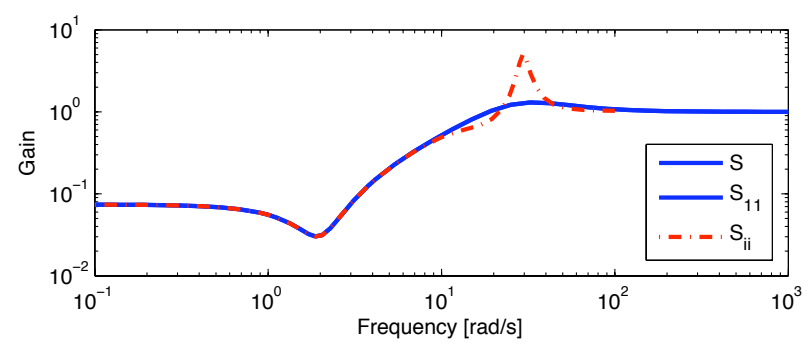

Fig. 3. Topology 1: sensitivity functions Bode plot.

that the disturbances on those frequencies will be amplified five times. Even if the system is stable, the interconnection has caused a loss of a significant part of the stability margins, which are now 2.3 for the gain margin and $17.5^{\circ}$ for the phase margin (Figure 4).

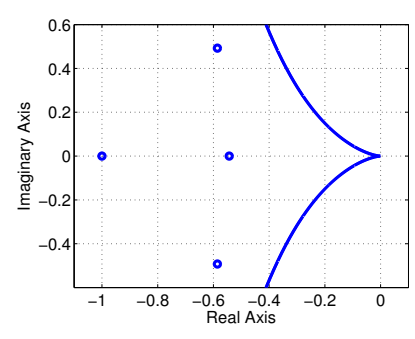

(a) Topology 1

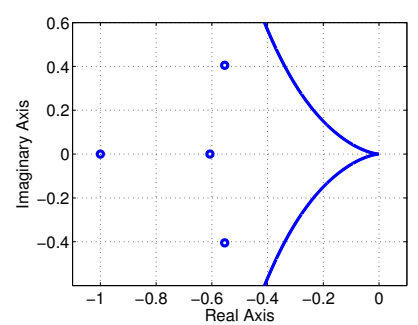

(b) Topology 2
Fig. 4. Nyquist plot and critical points.

Looking at topology 2 we notice that it is exactly like topology 1 but with arc 12 added. This added arc creates a new cycle and transforms the graph into a strongly connected one. Even if only one arc is added and the critical points of topology 2 do not move too far from the ones of topology 1 (see Figure 4), the sensitivity transfer functions in Figure 5 are very different from Figure 3. Comparing Figure 5 with Figure 3 we can see that the disturbance attenuation for low frequencies is worse than in the leader-follower case, having attenuation factors of about 0.3 and 0.2 , but near the cut-off frequency the peaks are lower $M_{s}<1.5$. As we expected, the poor behavior at low frequencies is given by nodes 2 and 3 because two cycles pass through them while only one cycle passes through nodes 1,4 and 5 . The stability margins 


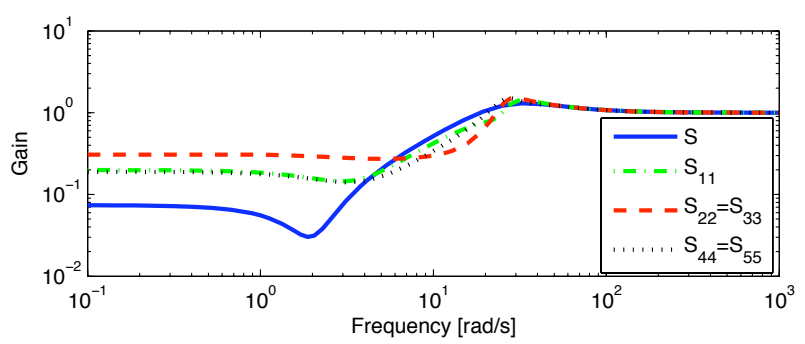

Fig. 5. Topology 2: sensitivity functions Bode plot.

for topology 2 are still reduced if compared to the single agent case, but they are better than for topology 1: 2.86 for the gain margin and $19.5^{\circ}$ for the phase margin.

What about the off-diagonal sensitivity functions? The low frequency behavior is similar to the corresponding diagonal sensitivity functions, while at high frequencies the gain drastically decreases. $\widetilde{S}_{i j}$ are band-pass filters and this can be seen in Figure 6, where the Bode plot of some off-diagonal sensitivity functions is shown.

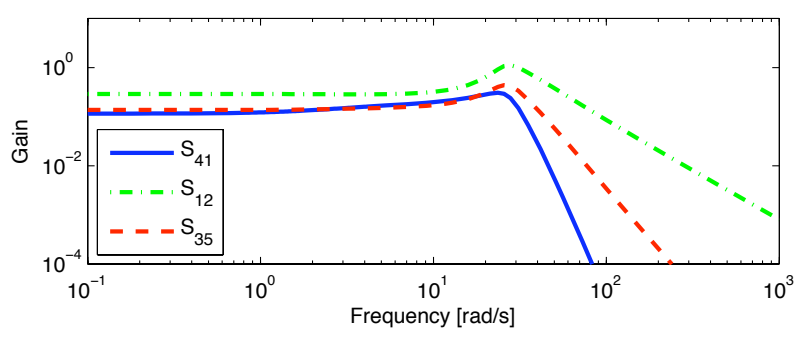

Fig. 6. Topology 2: Bode plot of some off-diagonal sensitivity functions.

\section{CONCLUSIONS AND FUTURE WORK}

The formula derived in Section IV shows how to compute the network sensitivity functions for arbitrary graphs and number of agents. We expect that this framework, together with the considerations in Section $\mathrm{V}$, will be helpful for multi-agent controller designers.

From the examples above we can conclude that the interconnection topology influences the sensitivity functions in two ways:

1) the cycles influence the low frequency behavior;

2) the Laplacian spectrum influences the peak value.

Given a topology, the open loop transfer function should have higher gain at low frequencies in order to better attenuate the disturbances. But because of the Bode's integral formula, a higher gain reduces the stability margins leading to a rise of the sensitivity function's peak.

No matter how the controller is designed, there are fundamental limitations to performance. Control with only feedback does not guarantee disturbance rejection. For this reason, a two degree of freedom controller is needed. In order to improve the properties of the multi-agent system, the feedforward compensation should filter the disturbances arriving from the agent's neighbors.
Our analysis demonstrates that the presence of cycles in the interaction topology degenerates the system's performance. Fax [10] arrived to a similar conclusion when observed that adding a link to a system caused a loss on the stability margin. If there are cycles in the graph, the disturbance entering on an agent passes through its neighbors and comes back making more difficult to attenuate it.

In this paper we have considered only systems with identical dynamics, but we expect that this approach can be extended to heterogeneous systems. We conjecture that polynomials of the network sensitivity functions will include different plant models, but that the paths and cycles structures will influence the performances in the same way.

\section{ACKNOWLEDGEMENTS}

The authors would like to thank Håkan Terelius and Anna Maria Cherubini for their feedback on the material in this paper.

\section{REFERENCES}

[1] J. Corfmat and A. Morse, "Decentralized control of linear multivariate systems," IEEE Transactions on Automatic Control, vol. 12, pp. 476495, 1976.

[2] D. Siljak, Decentralized control of complex systems. Boston, MA. Academic Press, 1991.

[3] M. Mesbahi and F. Hadaegh, "Formation flying control of multiple spacecraft via graphs, matrix inequalities, and switching," Journal of Guidance, Control, and Dynamics, vol. 24, no. 2, pp. 369-377, 2001.

[4] C. Langbort, R. Chandra, and R. D'Andrea, "Distributed control design for systems interconnected over an arbitrary graph," IEEE Transactions on Automatic Control, vol. 49, no. 9, pp. 1502-1519, 2004.

[5] D. Zelazo, A. Rahmani, and M. Mesbahi, "Agreement via the edge laplacian," in Decision and Control, 2007 46th IEEE Conference on, 2007, pp. 2309-2314.

[6] D. Zelazo, A. Rahmani, J. Sandhu, and M. Mesbahi, "Decentralized formation control via the edge Laplacian," in American Control Conference, 2008, 2008, pp. 783-788.

[7] F. Borrelli and T. Keviczky, "Distributed LQR design for identical dynamically decoupled systems," IEEE Transactions on Automatic Control, vol. 53, no. 8, pp. 1901-1912, 2008.

[8] P. Massioni and M. Verhaegen, "Distributed control for identical dynamically coupled systems: a decomposition approach," IEEE Transactions on Automatic Control, vol. 54, no. 1, pp. 124-135, 2009.

[9] Z. Jin and R. M. Murray, "Double-graph control strategy of multivehicle formations," in 43rd IEEE Conference on Decision and Control, 2004. CDC, vol. 2, 2004.

[10] J. A. Fax and R. M. Murray, "Information flow and cooperative control of vehicle formations," IEEE Transactions on Automatic Control, vol. 49, no. 9, pp. 1465-1476, 2004.

[11] S. Tonetti and R. M. Murray, "Limits on the network sensitivity function for multi-agent systems on a graph," California Institute of Technology, Pasadena, CA, Tech. Rep., 2009.

[12] B. Bollobás, Modern graph theory. Berlin. Springer, 1998.

[13] F. Chung, Spectral graph theory. American Mathematical Society, 1997.

[14] S. Mason and H. Zimmermann, Electronic circuits, signals, and systems. Wiley New York, 1960.

[15] S. Mason, "Feedback theory-some properties of signal flow graphs," Proceedings of the IRE, vol. 41, no. 9, pp. 1144-1156, 1953.

[16] — "Feedback theory-further properties of signal flow graphs," Proceedings of the IRE, vol. 44, no. 7, pp. 920-926, 1956.

[17] H. Bode, Network analysis and feedback amplifier design. D. Van Nostrand, Princeton, NJ, 1945. 